\section{Probenvorbereitung, für Atomspektrometrie}

J. Knecht

FB Chemie; Division.:Analytical Chemistry, Universität

Marburg, Marburg, Deutschland

Englischer Begriff sample preparation for atomic spectrometry

Definition Umfasst alle Maßnahmen, um die Proben für die atomspektrometrische Messung vorzubereiten ( $\triangleright$ Atomspektrometrie).

Beschreibung Es ist zwar möglich, einige Elemente direkt aus der zu untersuchenden Lösung mit atomspektrometrischen Verfahren zu bestimmen, wenn an die Qualität der Analysenergebnisse nicht die höchsten Anforderungen gestellt werden, aber im Allgemeinen muss die Probe vor der Messung aufbereitet werden. Dies kann entweder geschehen durch Trockenveraschung (= Erwärmen der Probe in einem Glühofen oder einer heißen Flamme) oder durch Nassveraschung (= Erhitzen mit oxidierenden Säuren oder mit Mischungen dieser Säuren). Neuerdings wird auch wegen der Zeit- und Kostenersparnis oft die Mikrowellenveraschung eingesetzt. Daneben gibt es eine ganze Reihe von Spezialveraschungsmethoden wie Plasmaveraschung etc.

Das Gebiet der Probenvorbereitung ist zu umfangreich, um hier ausführlich behandelt zu werden. Dafür muss Spezialliteratur herangezogen werden.

\section{Literatur}

Bock R (2001) Handbuch der analytisch-chemischen Aufschlussmethoden. Wiley-VCH, Weinheim 